\title{
A INTEGRAÇÃO ENTRE BIM E GIS COMO FERRAMENTA DE GESTÃO URBANA ${ }^{1}$
}

\author{
INTEGRATION OF BIM AND GIS: A TOOL FOR URBAN \\ MANAGEMENT \\ Fernando Almeida \\ Departamento de Arquitetura e Urbanismo, Mestrado em Desenvolvimento Urbano, UFPE \\ f@fernandoalmeida.arq.br \\ Max Andrade \\ Departamento de Expressão Gráfica, Mestrado em Desenvolvimento Urbano, UFPE \\ maxandrade73@gmail.com
}

\begin{abstract}
RESUMO
Cada edifício tem sua contribuição individual e mensurável no consumo de recursos, geração de resíduos e impacto na vizinhança em uma cidade. Este artigo busca investigar preliminarmente como a interseção entre BIM (dados dos edifícios) e GIS (dados da cidade) pode vir a contribuir na criação de um sistema integrado. Servirá a concessionárias públicas e a órgãos de gestão urbana para controlarem com maior precisão áreas de saturação e risco, assim como avaliar parâmetros de sustentabilidade em diversas escalas. Este sistema também atenderá aos projetistas e construtores, ao permitir que dados atualizados das infraestruturas públicas subsidiem a elaboração dos novos projetos. Pretende-se apresentar uma discussão teórica exploratória sobre os modelos vigentes e possibilidades de integração por meio de uma análise preliminar das similaridades e divergências semânticas dos sistemas GIS e BIM, e, para tal, parte-se de uma revisão bibliográfica acerca de iniciativas em experimentação nessa área que almejam mitigar os entraves que tornam o processo de integração de informações moroso, com elevado grau de inconsistência e difícil manipulação/visualização.
\end{abstract}

Palavras-chave: TIC. BIM. GIS. Semântica. Gestão urbana.

\begin{abstract}
Every building has its individual and measurable role on resources consumption, waste generation and neighborhood impact within a city. This paper preliminarily investigates how BIM (building data) and GIS (city data) intersection can contribute in creating an integrated system to attend public utilities and urban management offices for addressing accurate control on saturated and under-risk areas, as well as evaluating sustainability parameters at various scales. This system will also attend designers and contractors, by enabling up-to-date public infrastructure network data to be accessed during the project phase. Aiming to present an exploratory and theoretical discussion of current models and integration possibilities among semantics of GIS and BIM systems, this work proceeds with a systematic review of ongoing research initiatives on the field that strive to mitigate integration barriers found on the process of unifying various data, be it time consuming practices, high level of inconsistency on data compilation and/or hard handling data visualization.
\end{abstract}

Keywords: TIC. BIM. GIS. Semantics. Urban management.

\footnotetext{
${ }^{1}$ ALMEIDA, F.; ANDRADE, M. A integração entre BIM e GIS como ferramenta de gestão urbana. In: ENCONTRO BRASILEIRO DE TECNOLOGIA DE INFORMAÇÃO E COMUNICAÇÃO NA CONSTRUÇÃO, 7. , 2015, Recife. Anais... Porto Alegre: ANTAC, 2015.
} 


\section{INTRODUÇÃO}

Redes de mobilidade saturadas, alagamentos, crises de fornecimento de energia e água, manejo ineficiente de resíduos sólidos, decadência das redes físicas urbanas, desequilíbrios microclimáticos, entre outros, são alguns dos eventos críticos que permeiam as grandes cidades e são resultantes, em sua grande parte, das inter-relações entre as infraestruturas urbanas e as edificações que the são contíguas (CONWAY-GOMEZ, 2009; KONRAD, 2014).

Estas inter-relações, entretanto, são passíveis de serem mensuradas e decodificadas em sistemas. No caso das infraestruturas urbanas, podem ser analisadas as redes de distribuição de água e energia, de captação de águas pluviais, de coleta de esgotos e de resíduos sólidos, as redes viárias de passeios e faixas de rolamento, arborização pública, etc. No caso das edificações, isto dá-se a partir de suas contribuições individuais de consumo de recursos (água e energia), geração de resíduos (esgoto e lixo), impactos na vizinhança (redução na permeabilidade do solo, aumento no fluxo pontual de veículos, emissão de ruídos), etc. Seja no âmbito urbano amplo ou das edificações isoladas, hoje dispõe-se de uma variada gama de ferramentas aplicadas de planejamento e gestão, fundamentalmente embasadas em recursos computacionais, as quais demonstram eficiência em atuar nos processos de mensuração, decodificação e registro dos dados processados.

Entre os recursos computacionais mais utilizados como instrumento eficiente de gestão da informação pública e de tomada de decisões destaca-se o Sistema de Informação Geográfica (SIG). O SIG, ou GIS (Geographic Information System), como é mais difundido, é uma base instrumental já bem estabelecida há décadas nos meios da produção geográfica, cartográfica e de planejamento urbano e regional. Trata-se, entre outras características, de uma base de dados digital de múltiplas finalidades, na qual um sistema de coordenadas espaciais em comum é o meio básico de referência. Em razão de aglomerar dados oriundos de bases textuais (dados tabulares) a bases gráficas georreferenciadas (mapas, fotos aéreas e afins), consagrou-se como um sistema altamente eficaz para tomada de decisões, ao permitir uma livre manipulação destes dados por meio de pesquisas (queries) e combinações variadas, sempre amparadas por uma representação gráfica, vetorial ou raster (FOOTE e LYNCH, 1995).

Da parte do edifício e sua construção, um processo computacional de gestão da informação importante que tem sido cada vez mais utilizado é o Building Information Modeling (BIM). $\mathrm{O}$ BIM partilha de semelhante princípio integrador e da consolidação de um robusto banco de dados, mas focado fundamentalmente no ciclo-de-vida da construção e na relação entre seus componentes. Muito empregado com a finalidade de aumentar a produtividade nas áreas de arquitetura, engenharia e construção (AEC), o BIM tem uma característica colaborativa que Ihe coloca como um processo de grande potencial de integrar com outras tecnologias (CRESPO; RUSCHEL, 2007; SUCCAR, 2013).

Este artigo é um recorte de uma pesquisa de mestrado, em desenvolvimento, na qual partese do pressuposto de que a integração entre o GIS e o BIM pode resultar em um sistema bidirecional capaz de permitir à gestão pública ter dados mais precisos sobre os coeficientes de consumo de recursos, geração de resíduos e impactos de vizinhança dos edifícios. E à gestão do empreendimento da construção permitir obter informações consistentes e atualizadas sobre dados públicos do cadastro territorial, condicionantes legais e recursos infraestruturais disponíveis. O objetivo deste sistema integrado é fundamentar a gestão pública na tomada mais precisa de decisões em questões de planejamento urbano, revisão legal e/ou gestão de crises urbanas e catástrofes, contribuindo com a iniciativa da construção civil em realizar projetos e obras com mais celeridade e mais equilibradas em relação ao ambiente no qual se inserem.

Entres as limitações encontradas durante a elaboração deste artigo, há destaque para a pouca produção bibliográfica sobre o assunto, particularmente a desenvolvida no Brasil, 
quando observada a ampla bibliografia dedicada ao GIS e ao BIM isoladamente. Tal qual a pouca representatividade da produção científica sobre o assunto, é semelhante a falta de casos no mundo onde a experimentação em si, por meio dos órgãos públicos, tenha sido realizada ou, ao menos, documentada. Nos casos das pesquisas, a predominância do assunto da integração entre GIS e BIM tem mais habitualmente como foco, da parte do GIS, a busca por um processo de representação tridimensional baseada no BIM, e, da parte do BIM, a capacidade de representar os dados da construção geoespacialmente.

Uma condicionante estabelecida para elaboração tanto da pesquisa como deste artigo é a premissa de que todo projeto de edificação em lote urbano deva ser validado por um órgão competente da municipalidade para que possa ser construído, cabendo a este órgão a retenção destas informações para a consolidação de um acervo e banco de dados próprio. Ainda que este acervo seja quase que totalmente mantido em meio físico (pranchas de desenhos e cadernos de textos), considera-se que esta documentação venha futuramente a ser substituída ou complementada por uma documentação digital em BIM.

O propósito deste artigo é levantar um acervo bibliográfico e alguns direcionamentos conceituais, por meio de uma discussão teórica exploratória, para fundamentar a integração entre o GIS e o BIM. Na pesquisa são investigados estes direcionamentos em pormenor, assim como avaliadas suas soluções tecnológicas e formas de adaptabilidade à realidade local, neste caso, na Cidade do Recife.

\section{CAMINHOS PARA UMA INTEGRAÇÃO SEMÂNTICA}

O grande potencial do BIM e do GIS, comprovado por diversos artigos publicados nos últimos anos (CRUZ; CAMPOS, 2005; OLIVEIRA, 2009; HERGUNSEL, 2011; NOUR, 2011) justifica a capacidade de se estabelecer uma integração da informação que circula entre ambos os processos de trabalho digital. A interação que se propõe discutir neste artigo dá-se pela integração da informação sobre os dados físicos e funcionais do edifício, extraídos de softwares de modelagem BIM, com os dados de âmbito urbano (inclusive socioeconômicos) contidos nas bases em GIS. Discute-se neste artigo, portanto, a viabilidade de consolidação de um sistema BIM/GIS capaz de estabelecer uma ampla rede de interações de informação entre a escala do edifício e a escala da cidade (Figura 1).

Figura 1 - Síntese das escalas tradicionais de aproximação em GIS e BIM

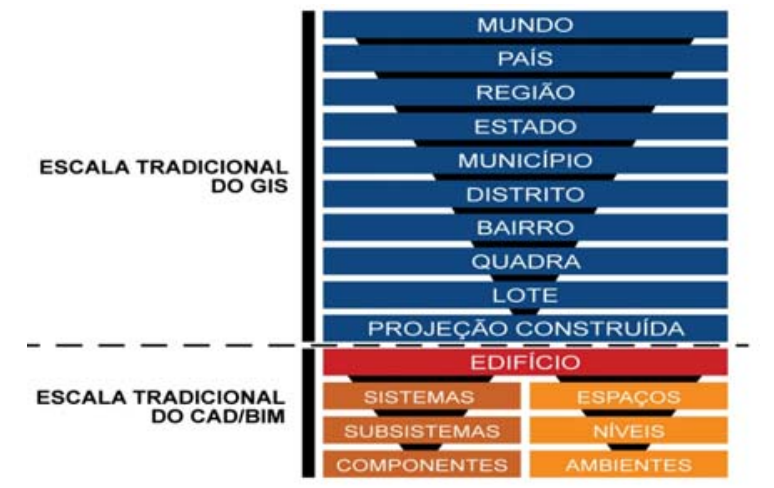

Fonte: Przybyla, 2010, adaptado e traduzido pelo autor.

O campo de aplicações da integração entre BIM e GIS é tão vasto que não é possível estabelecer um procedimento padrão comum de integração que seja consenso internacional. Várias iniciativas ao redor do mundo tem assumido caminhos distintos na integração da informação, em razão de suas necessidades peculiares (PRZYBYLA, 2010). Uma unanimidade, entretanto, é a busca por uma universalização dos formatos dos arquivos 


\section{1}

digitais, e, neste sentido, o GIS tem a grande vantagem de se utilizar de um formato de dados espaciais (o shapefile) o qual é um formato aberto desde a sua origem.

Um shapefile consiste basicamente em um arquivo principal ( ${ }^{*}$.shp), um arquivo-índice (*.shx) e uma tabela $d B A S E\left({ }^{*} . d b f\right)$, havendo ainda outros arquivos acessórios que também podem estar associados a um mesmo shapefile. $\mathrm{O}$ arquivo principal é um arquivo de acesso direto no qual cada um representa um conjunto de dados de uma mesma natureza de forma (ponto, linha ou polígono) com uma lista de seus vértices. O arquivo-índice tem a finalidade de indexar a leitura do arquivo principal, de modo a permitir que o software faça buscas rápidas no conteúdo deste. A tabela $d B A S E$ contém todos os atributos literais atribuídos às feições presentes naquele arquivo principal (FOOTE; LYNCH, 1995; ESRI, 1998).

No que toca ao BIM, em razão da maior complexidade técnica do seu conteúdo digital, há uma grande variedade de formatos de arquivos, proprietários e livres. Entretanto, em 1994 deu-se início ao desenvolvimento de um arquivo padrão aberto para o BIM, o Industry Foundation Classes (IFC), com a finalidade de garantir o máximo de interoperabilidade entre as diferentes instâncias envolvidas no processo da construção (LAAKSO; KIVINIEMI, 2012). É constituído por um único arquivo de extensão *.ifc, e a linguagem de sua codificação permite que ele consiga ser ao mesmo tempo compacto e bem estruturado para suportar variadas regras de validação de dados. Sua mais recente versão, o IFC4 de 2013, incorpora várias extensões do IFC para a construção, serviços e estruturas, geometrias avançadas e vários outros recursos, inclusive alguma interoperabilidade entre BIM e GIS (LIEBICH, 2013; BUILDINGSMART, 2015).

Em razão do BIM valer-se da representação 3D como a sua expressão visual mais expoente, - GIS apenas recentemente veio ser compatível com uma geometria tridimensional (PRZYBYLA, 2010), o que abriu novos caminhos para a integração entre a base de dados georreferenciada e uma interface baseada na visualização tridimensional (DÖLLNER; HAGEDORN, 2008).

Operacionalmente, o modelo BIM foca na geometria sólida construtiva, na representação de limites, em operações Booleanas, e sua modelagem de dados é baseada em arquivo (filebased). Uma característica importante do BIM é a decomposição e especialização dos objetos dentro de um modelo, bem como a relação entre eles. Em contrapartida, o GIS tem uma forte vocação em georreferenciação, de modo que a relação entre seus objetos é baseada em coordenadas. Sua modelagem de dados é baseada em servidor (server-based), e dispõe de recursos que permitem realizar de forma bastante simples e rápida análises de sobreposição (uniões, interseções), de proximidade (buffer, distância entre pontos), de superfície (aspectos topográficos, mapas de inclinações), de roteamento (redes de transporte, de fluidos), temporais e estatísticas (BERLO; LAAT, 2011).

Com o intuito de suprir esta demanda do BIM por uma base de dados unificada foram criadas diferentes ferramentas compartilhadoras de modelos, seja entre aplicativos, seja a partir de compartilhamento em banco de dados centralizados ou banco de dados federativos (RUSCHEL et al., 2013). Entre estas ferramentas destaca-se o BIM Server, o qual trata-se de um serviço open source elaborado para exercer a função de servidor de arquivos e permitir que os dados sejam interpretados e armazenados como objetos em um banco de dados central. A grande vantagem deste recurso é permitir que os dados BIM sejam facilmente inquiridos, mesclados e filtrados, tal qual acontece no GIS, mas em um ambiente nãoproprietário (BIMSERVER, 2015).

A aproximação entre BIM e GIS tem sido investigada por vários pesquisadores ao redor do mundo. Na predominância dos casos, o interesse é trazer o ferramental do GIS para a área da gestão de empreendimentos, ou Facility Management (FM), como é mais difundido. Esta é uma abordagem mais fortemente ligada ao BIM, em razão da necessidade de uso extensivo de dados da edificação, seja à escala dos componentes construtivos (manutenção de 
elementos da construção) ou dos seus níveis e usos (integração com dados de recursos humanos, sistemas de segurança etc.). O emprego do GIS nestes casos recai na aplicação de suas ferramentas analíticas e pela sua capacidade de integrar dados de bases distintas (SEBASTIAN et al., 2013). Estudos mais aprofundados sobre o estado-da-arte deste assunto demonstram que as tentativas de integração entre BIM e GIS ora recaem mais para o processo do BIM (maior foco no edifício, em razão do já adquirido acúmulo de informações sistematizadas), ora para o do GIS (maior foco geoespacial regional, integrando sua base de dados a alguns dados superficiais extraídos do modelo BIM) (BERLO; LAAT, 2011).

Um dos primeiros esforços em integrar modelos IFC (BIM) e GIS com a finalidade de atender a uma demanda do âmbito da gestão urbana foi o IFG (IFC For GIS), patrocinado pela BuildingSMART International, e elaborado pela Norwegian State Planning Authority com o propósito de estabelecer uma base geográfica e de construções dentro de uma estrutura de dados consistente e que servisse de suporte para sistemas de validação de conformidade de códigos legais de construção e de edificações (HIJAZI et al., 2009). O IFG permite a representação de parcelamento do solo, de elementos geométricos construídos, mapas, sistemas de coordenadas, contornos, redes e sistemas de distribuição (água, energia e esgoto), relações de proximidade, dados de pesquisas de campo, relevo, identificações semânticas dos edifícios e elementos construtivos (edifícios, paredes, janelas, portas, aberturas) (KARIMI; AKINCl, 2009). O objetivo final seria dar mais celeridade aos processos de aprovação de projetos, associando as informações preexistentes de um banco de dados central GIS com as informações de registro de propriedade advindas das bases AEC/FM (BIM). Assim, os projetistas e construtores teriam acesso a um conjunto de informações georreferenciadas referentes ao cadastro do terreno, ao zoneamento, às redes públicas de serviços disponíveis etc. (CITYGML-WIKI, 2006). Da mesma forma, os órgãos da gestão pública teriam vários usos possíveis para este sistema, deste a gestão de crises como a manutenção do uso do solo (KARIMI; AKINCI, 2009).

O IFG, em suma, se caracteriza por se basear na semântica do modelo IFC, com o diferencial de incorporar informações necessárias para dar aos empreendimentos o contexto espacial necessário para a tomada de decisões baseada em uma localização geográfica. Apesar de ter um escopo relativamente estreito, é uma demonstração de uma integração possível de ser realizada, mas sem a pretensão de explorar o potencial máximo do GIS e do BIM (PRZYBYLA, 2010).

Esta preocupação em estabelecer um procedimento mais eficiente e preciso para a aprovação de projetos, entretanto, já havia sido lançada em 1995 em Singapura, onde foi desenvolvido um sistema próprio de validação de modelos digitais BIM, em uma época na qual mal se conhecia uma alternativa ao CAD. Este sistema, em vigor ainda hoje e chamado CORENET (Construction Real Estate Network), consiste em um sistema baseado na internet (webbased), do tipo G2B (Government to Business, ou seja, mantido pelo Governo e direcionado às iniciativas privadas) com a finalidade de permitir aos profissionais das áreas de arquitetura, engenharia e construção (AEC) submeterem projetos por meio eletrônico para aprovação e análise de conformidade junto às autoridades competentes (KHEMLANI, 2005; AUTHORITY, 2009).

Esta iniciativa é certamente a mais ambiciosa de estímulo à prática do BIM já desenvolvida por um órgão público, o que tem sido responsável por grandes mudanças tecnológicas da parte dos profissionais de AEC em Singapura, além do aumento da transparência da parte do governo (LIN; FATT, 2006). Entretanto, o CORENET é uma ferramenta exclusivamente dedicada ao ambiente BIM das construções, não tendo nenhuma relação direta com as infraestruturas urbanas, ou mesmo com o GIS.

Com o intuito de explorar potencial de integração entre BIM e GIS ao seu limite, um grupo com mais de 70 empresas, instituições de pesquisa e prefeituras da Alemanha foi criado com 
a finalidade de desenvolver e explorar a visualização georreferenciada de modelos interoperacionais tridimensionais, o CityGML. Este modelo de informação tem sua base de codificação em XML (uma linguagem aberta desenvolvida para permitir um compartilhamento de dados com fácil leitura tanto por humanos como por máquinas) e consiste em um modelo e um mecanismo padrões para descrever objetos tridimensionais em relação às suas geometrias, topologias, semânticas e aparências, definindo cinco níveis de detalhamento, ou Levels of Detail (LoD) (KOLBE, 2012). Ainda assim, ao contrário de constituir-se de uma semântica exclusiva, o CityGML tem uma maior aproximação com a linguagem e lógica do GIS, o que the valeu o reconhecimento e oficialização como formato internacional padrão e aberto (open source) para troca de dados espaciais pelo Open Geospatial Consortium (OGC) (CONSORTIUM, 2015).

Outra importante característica do CityGML é a sua extensibilidade, o que permite que sejam criadas extensões com as mais variadas finalidades, favorecendo a existência, por exemplo, de mais meios de se possibilitar a conversão de dados de uma base IFC para a CityGML/GIS, oriundos de outras fontes de dados (CITYGML-WIKI, 2012). Apesar desta flexibilidade, ainda não foi desenvolvida uma atualização do CityGML ou uma extensão que possa lidar com as redes públicas de serviço (sistemas de dutos, redes técnicas, etc.)(DÖLLNER; HAGEDORN, 2008).

\section{O BIM E SEU IMPACTO URBANO}

Constitui-se como parte integrante de um projeto arquitetônico executivo a previsão do consumo de água, de energia elétrica e emissão de resíduos líquidos, sem os quais não é possível dimensionar os reservatórios, quadros de distribuição e fossas/dutos de escoamento. A partir de um leiaute prévio da ocupação de uma residência projetada, alguns parâmetros são direta ou indiretamente definidos, tais como consumo médio de água por pessoa e por dia (e proporcional despejo de águas cinza e negra), consumo de energia, etc.

A inserção de ferramentas de CAD no processo de projeto pouco mudou esta prática de dimensionamentos, exceto pelo aumento da precisão da sua estimativa. Mas estes dados mantem-se atrelados a um desenho bidimensional sem "inteligência própria", o que pode ser entendido por um computador apenas como linhas de texto. São, portanto, representados em memoriais descritivos desenvolvidos em processadores de texto, ou em tabelas não integradas, as quais resultam em anexos destes mesmos documentos de texto. Em suma, da prática difundida, mesmo com o uso do CAD, os dados de desempenho do edifício, particularmente os parâmetros de consumo hídrico e energético e emissão de resíduos líquidos, não são aproveitados como dados paramétricos digitais.

Com a gradativa implementação do BIM, o fundamento de se operar com elementos parametrizáveis e de se poder atribuir dados qualificados a um modelo tridimensional estabelece um novo paradigma na gestão da informação da construção, permitindo que várias ferramentas de análise de desempenho sejam utilizadas com eficiência (ADDOR et al., 2010). Assim, os dados relativos às estimativas de consumo de recursos e emissão de resíduos podem estar integrados ao modelo digital e o controle das informações sobre os impactos da edificação no sítio tende a tornar-se cada vez mais acessível.

Nos locais onde o BIM já é bastante difundido, é possível, durante o projeto, conceber um edifício energeticamente mais eficiente e econômico (por meio de especificações precisas de componentes economizadores e pela otimização do desempenho energético, em virtude do uso da simulação computacional ainda nas etapas iniciais do projeto) e, durante as obras, reduzir sensivelmente o desperdício de materiais de construção (UTIOME et al., 2014). O acompanhamento da pós-ocupação da edificação por meio do BIM pode permitir, por exemplo, monitorar e reduzir as emissões de $\mathrm{CO}_{2}$ e o consumo energético do edifício ao longo do tempo (MOTAWA; CORRIGAN, 2012). 
Ao analisarmos o desempenho das construções de uma maneira coletiva, ou seja, quando um conjunto de modelos BIM que caracterizam uma vizinhança são avaliados como um somatório, a noção de desempenho também se expande para a vizinhança, tornando assim possível interpretar de uma maneira holística e precisa o grau de impacto das edificações em uma região. A centralização desta análise, por sua vez, depende um órgão gestor público que disponha de um sistema que processe estes dados de desempenho junto a outros dados advindos de outras fontes (concessionárias públicas, dados censitários etc.) e permita diversas análises. Nesta etapa, evoca-se o GIS como um aclamado sistema para manipulação deste tipo de informação (SMARTCITIES, 2011).

\section{O GIS COMO PARADIGMA DA GESTÃO URBANA}

O aumento da complexidade das relações entre o ambiente construído das cidades e seu parcelamento tem levado aos órgãos de gestão urbana a condições críticas de operação, basicamente pela dificuldade em manipular um crescente volume de informações (DUARTE, 2010). Com o advento e difusão do projeto assistido por computador, houve um relativo avanço no sentido de incorporar estas questões em sistemas computacionais, possibilitando que um grande volume de dados pudesse ser coletado, processado, recuperado e analisado com rapidez e confiabilidade. Entretanto, é notável que boa parcela dos investimentos nas tecnologias computacionais tem sido direcionadas ao controle de processos administrativofinanceiros, em detrimento dos processos de gestão urbana e de políticas públicas (OLIVEIRA; OLIVEIRA, 2005).

Como solução tecnológica, o GIS já tem sido empregado neste tipo de demanda em diversas partes do mundo desde o início dos anos 70 do século passado, e da sua concepção aos dias atuais grandes avanços foram conquistados, especialmente no que se refere à viabilidade econômica para a aquisição tanto de softwares como de hardwares, o que o tem popularizado. O que se nota no Brasil, todavia, é que o GIS ainda não corresponde a uma prática padrão, sendo substituído, involuntariamente, por uma combinação de outros sistemas de informação digital (CAD, planilhas eletrônicas, documentos de texto). Isto acontece principalmente em função da formação e prática profissional daqueles que compõem o corpo técnico das áreas de planejamento e gestão nas administrações municipais, em boa parte engenheiros, arquitetos e urbanistas. A falta de uma padronização nos procedimentos para a utilização de bases de dados distintas é um dos fatores que pode dificultar o uso dessas ferramentas computacionais na gestão pública. Além do mais, o emprego de um grande número de softwares requer uma complexa rotina de manutenção de versões e compatibilidades (ORTH; SILVA, 2010).

Além desta questão de habilidade técnica, a realidade mostra que a maioria dos municípios brasileiros tem seus dados e informações sobre o território de forma setorizada, desarticulada e não georreferenciada. A necessidade de se deter a localização geográfica de alguns dados é relevante no processo de gestão territorial porque permite verificar como estes dados se distribuem no espaço, possibilitando, assim, conhecer as limitações e potencialidades territoriais (ORTH; SILVA, 2010).

\subsection{O GIS na Cidade do Recife}

A pesquisa da qual este artigo é um recorte inicial aborda a Cidade do Recife como caso de contexto urbano, em razão de sua recente investida em aperfeiçoamento de sua base tecnológica de informação cartográfica. Em 2006, a Prefeitura do Recife deu início a um processo de mapeamento digital com enfoque na modernização do seu sistema cartográfico, o que representou uma gradativa mudança de paradigma quanto ao seu modelo operacional, aderindo maciçamente ao uso de GIS. Planejado para ser alimentado gradativa e continuamente por informações de algumas secretarias municipais, foi posteriormente 
convertido em uma plataforma web com livre acesso para o grande público, chamado E-SIG (SILVA; NEVES, 2012).

Este sistema, concebido internamente para disseminar informações georreferenciadas na Administração Municipal, tem como principal funcionalidade servir de base de endereçamento para todos os serviços da Prefeitura, com informações dos logradouros públicos municipais dentro de uma codificação única. Entre os dados gerais disponíveis no sistema web encontram-se bairros, quadras, lotes, arruamentos e alguns equipamentos públicos (RECIFE, 2010).

O banco de dados do E-SIG está disponível no site para download em formato shapefile, e, por meio do uso de software de geoprocessamento, é possível acessar as bases de informação que fundamentam as feições gráficas que the compõem. Na camada (ou shapefile) de "Lotes", por exemplo, é possível saber, de cada lote, a sua área, a área total construída, a quantidade de pavimentos, o ano de construção, entre outros dados (ver Figura 1). Da mesma forma, é possível filtrar a exibição do conteúdo vetorial de acordo com as informações da tabela que Ihe está atrelada. Um exemplo é a possibilidade de atribuir uma escala de cores para exibir um grupo de intervalos numéricos, o que pode ser aplicado para observar as manchas das áreas com maior ou menor gabarito (extraídas do atributo QTDPAVIMEN), ou das áreas com os edifícios mais antigos (extraídas do atributo ANCONSTR).

Figura 1 - Tabela de atributos da camada "Lotes", extraída da base do E-SIG

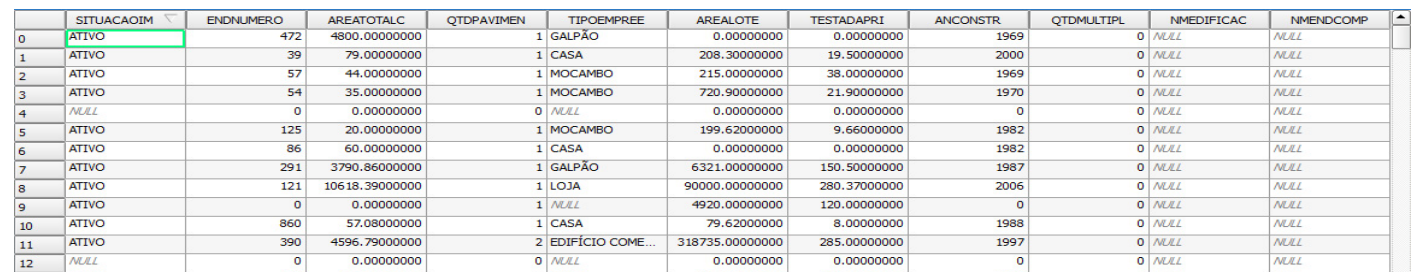

Fonte: Almeida, 2015.

O grau de detalhamento da base de dados digital georreferenciada e sua tabela de atributos é diretamente proporcional ao grau potencial de tomada de decisão, pois as feições individualmente podem expressar graficamente um número praticamente ilimitado de atributos (YEH, 1999). A inserção de dados extraídos do modelo BIM nesta tabela é um exemplo de como esta base pode ser incrementada, adicionalmente às demais fontes públicas. Em razão desta base já estar consolidada pela Prefeitura, a pesquisa vale-se deste banco de dados para desenvolver seu modelo experimental de integração BIM/GIS, a ser discutido em estágios mais avançados desta pesquisa.

\section{O POTENCIAL DA INTEGRAÇÃO}

A integração entre BIM e GIS, conforme observado anteriormente, direciona-se para mais de um caminho semântico: ora pende para o processo de trabalho baseados no BIM, ora pende para o processo de trabalho baseados do GIS. Posto que o foco da pesquisa e deste artigo, especificamente, esteja na gestão urbana, há um indicativo que o processo que se está em busca esteja mais fundamentado nas práticas do GIS, inclusive por ser a ferramenta com maior tendência de aplicação nos órgãos públicos de gestão.

Por outro lado, vê-se que esta integração tende a aproximar o BIM do discurso das infraestruturas públicas, corroborando, assim, para a difusão do uso do BIM nos projetos e obras das redes de infraestruturas urbanas (por exemplo, redes elétricas e hidráulicas públicas sendo planejadas por softwares MEP/BIM). Esta nova prática pode ser positiva no 


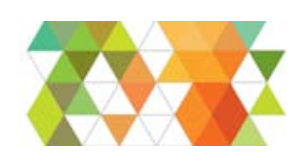

\section{TIC2015}

que se refere à integração das redes infra estruturais públicas (extra-lotes) e privadas (intralotes). Para a iniciativa privada, oferece a informação precisa sobre as especificações das redes públicas disponíveis e para as concessionárias de serviços públicos oferece a possibilidade de compatibilização automática da infraestrutura e a noção precisa do consumo das demandas, a partir da informação extraída diretamente do modelo BIM sobre a demanda dos empreendimentos. Além disto, outras vantagens do uso do BIM pelas concessionárias e órgãos de serviços públicos são: deter maior controle da execução e manutenção das redes instaladas; fundamentar um banco de dados que permita subsidiar de forma mais exata e preventiva revisões de regulamentos urbanos de uso e ocupação do solo; permitir tomadas de decisão mais embasadas mediante situações de crise.

Um exemplo desta integração é o cruzamento de uma camada de linhas da rede de esgotamento sanitário (com atributos de extensão, ano de implantação, bitolas dos dutos etc.) com a camada de linhas da testada do lote (com atributos de código cadastral, emissão média de esgoto, quantidade de famílias residentes etc.), obtendo uma representação das áreas mais ou menos saturadas na geração de resíduos líquidos. Esse mapeamento pode repercutir em um planejamento mais eficiente da manutenção das redes. Além do mais, pode resultar em previsões mais precisas dos pontos de saturação, o que pode influenciar no processo de aprovação de novos projetos em virtude da constatação "em tempo real" dos impactos dos mesmos nos índices de emissão. Em suma, as possibilidades de emprego dos recursos de GIS são variadas e o fator que irá atribuir sucesso a qualquer empreitada neste sentido será o rigor na aquisição, compilação e integração destes dados com outras bases.

O recorte metodológico da pesquisa concentra-se basicamente em três parâmetros obtidos a partir de uma base de dados das edificações: o consumo de água, de energia elétrica e o volume de resíduos líquidos emitidos. Desta forma será possível ter a amostra de um determinado espectro dos possíveis impactos dos empreendimentos no entorno e avaliar a capacidade de uma determinada área urbana de suportar os impactos de suas condições atuais e a de novos empreendimentos.

A escolha desses três parâmetros foi considerada para delimitar o escopo da pesquisa, mas também são considerados importantes outros parâmetros computáveis das edificações, tais como dimensionamento das tubulações de ligação com as redes públicas, volume estimado de resíduos sólidos gerados, efeito térmico da volumetria e materiais das fachadas, geração de fluxo de entrada e saída de veículos, entre outros.

Outro ponto a ser considerado é que métodos convencionais de avaliação dos impactos ambientais no/do edifício costumam estudar as construções como volumes isolados, ou apenas cercados das edificações imediatamente vizinhas, o que resulta em avaliações pouco consistentes, deixando de avaliar os reais impactos do empreendimento no contexto urbano. Todavia, num cenário em que as informações estejam integradas, especialmente se advindas de uma base BIM, é possível extrair informações mais precisas sobre a influência do entorno no desempenho do edifício e o impacto do volume do edifício no entorno, tornando mais consistentes os estudos de desempenho energético de uma região, os efeitos de suas ilhas de calor, sua pegada de carbono, consumo de água, transporte, rotas de fuga em emergências, etc. (MCROBERTS, 2010)

Este cenário, entretanto, é razoavelmente hipotético, se for considerado que os vários lotes que compõem uma quadra ainda serão construídos e que todas as construções estejam modeladas em BIM. Mas em uma condição mais próxima do real, propõe-se que haja uma integração entre as informações advindas das novas construções, tradicionais e/ou sistematizadas em BIM (com dados de previsão de consumo e emissão incorporados em seu modelo virtual), com as informações oriundas das edificações existentes (extraídas dos dados constantes nas contas dos serviços das concessionárias públicas). Dentro desse modelo integrado será possível estabelecer estimativas muito mais precisas sobre os recursos 
disponíveis e a emissão de resíduos, tornando possível manter um elevado nível de utilidade e precisão.

Das iniciativas de integração levantadas neste artigo, muitas informações ainda são necessárias para melhor se apreender tanto do mecanismo de criação das bases de dados como das relações semânticas de integração BIM/GIS. No caso do CORENET, por exemplo, por ser uma solução proprietária, pouco há publicado acerca de detalhes sobre seu funcionamento, de modo que a obtenção de maiores informações está sendo feita através da comunicação com os desenvolvedores. A mesma necessidade de contato com os desenvolvedores acontece no caso do IFG, cujo desenvolvimento foi concluído em 2007 e há pouca informação disponível desde então. No caso do CityGML, apesar de ser uma solução aberta, ainda não foi desenvolvido um modo de representação das redes de infraestruturas urbanas, o que abre possibilidades para que esta lacuna seja tratada em atualizações futuras ou pelo desenvolvimento de extensões.

\section{CONSIDERAÇÕES FINAIS}

A gestão pública do território urbano deve estar plenamente capaz de acompanhar as transformações que acontecem na cidade, com destaque para os efeitos da construção de novos edifícios em somatório com os preexistentes. Esta tarefa é nitidamente dependente da implementação bem-sucedida de um sistema tecnológico de monitoramento e análise. À medida que antigos processos (construção civil, por exemplo) incorporam novos mecanismos de desenvolvimento, fundados em bases digitais, é de se esperar que as municipalidades estejam preparadas para manipular estas informações. Em virtude dos recursos tecnológicos hoje disponíveis, é possível, inclusive, ir além da mera manipulação passiva de informações e promover a criação de uma rede mais ampla de dados, integrada tanto a estas novas tecnologias quanto aos métodos tradicionais de inserção de dados.

A revisão bibliográfica realizada para este artigo demonstra que, apesar de haver iniciativas consistentes acerca da associação dos modelos construtivos ao contexto urbano, ainda há uma ausência de estudos sobre a articulação entre BIM e GIS voltada ao planejamento e gestão urbanos. Apesar de se acreditar esta articulação deva ter um efeito de mão dupla, ou seja, beneficiar tanto a gestão pública quanto os projetistas e construtores com informações consistentes, observou-se que a iniciativa da parte de órgãos do governo é fundamental para que este processo tenha sustentabilidade, em razão de que lhe cabe definir parâmetros comuns de exigências legais e boas práticas.

O conjunto de informações levantadas neste artigo também demonstra que muito do que se alcançou como soluções tecnológicas partiu geralmente de demandas reais regionais (como Singapura, Noruega, Alemanha), e que algumas posteriormente avançaram rumo a uma padronização global. Este perfil regional é um fator relevante, pois é imprescindível haver uma sinergia entre uma solução padrão e a prática tecnológica regional. Portanto, reforça-se aqui a escolha da Cidade do Recife e sua base tecnológica em GIS como referência de caso para o desenvolvimento da pesquisa.

\section{REFERÊNCIAS}

ADDOR, M. R. A. et al. Colocando o "i" no BIM. ARQ.URB. São Paulo: USJT: 104-115 p. 2010.

AUTHORITY, B. C. Corenet e-Submission System FAQ. Singapore, 2009. Disponível em: < http://www.corenet.gov.sg/integrated submission/esub/esub faqs.html\#FAQ final 11 >. Acesso em: 21/02/2015.

BERLO, L. V.; LAAT, R. D. Integration of BIM and GIS: The development of the CityGML GeoBIM extension. In: KOLBE, T. H.;KÖNIG, G., et al (Ed.). Advances in 3D Geo-Information Sciences: Springer Berlin Heidelberg, 2011. 
BIMSERVER. About BIMServer. 2015. Disponível em: < http://bimserver.org/about/ >. Acesso em: 10/03/2015

BUILDINGSMART. IFC Overview summary. 2015. Disponível em: < http://www.buildingsmarttech.org/specifications/ifc-overview >. Acesso em: 13/03/2015.

CITYGML-WIKI. IFC for GIS. 2006. Disponível em: < http://www.citygmlwiki.org/index.php/IFC for GIS >. Acesso em: 09/03/2015.

CityGML GeoBIM ADE. 2012. Disponível em: < http://www.citygm/wiki.org/index.php/CityGML GeoBIM ADE >. Acesso em: 09/03/2015.

CONSORTIUM, O. G. CityGML. 2015. Disponível em: < http://www.opengeospatial.org/standards/citygml >. Acesso em: 20/03/2015.

CONWAY-GOMEZ, K. How does urban development affect the quality and quantity of natural resources? Population and Natural Resources case study, 2009. Disponível em: < http://cgge.aag.org/PopulationandNaturalResources1e/CS US July09/CS US July09 print.html >. Acesso em: 10/02/2015.

CRESPO, C. C.; RUSCHEL, R. C. Ferramentas BIM: um desafio para a melhoria no ciclo de vida do projeto. TIC2007 - III Encontro de Tecnologia de Informação e Comunicação na Construção Civil. Porto Alegre, RS: 1-9 p. 2007.

CRUZ, I.; CAMPOS, V. B. G. Sistemas de Informações Geográficas aplicados á análise espacial em transportes, meio ambiente e ocupação do solo. Rio de Transportes III. Rio de Janeiro, Brasil 2005.

DÖLLNER, J.; HAGEDORN, B. Integrating urban GIS, CAD, and BIM data by service-based virtual 3D city models. Leiden, The Netherlands: Taylor \& Francis/Balkema, 2008.

DUARTE, R. M. Geoprocessamento no Planejamento Urbano. InfoGEO. Curitiba, Brasil: MundoGEO Ltda. 2010.

ESRI. ESRI Shapefile Technical Description. Redlands, USA, p.1-34. 1998

FOOTE, K. E.; LYNCH, M. Geographic Information Systems as an Integrating Technology: Context, Concepts, and Definitions. Boulder, USA, 1995. Disponível em: <

http://www.colorado.edu/geography/gcraft/notes/intro/intro.html >. Acesso em: 13/01/2015.

HERGUNSEL, M. F. Benefits of Building Information Modeling for construction managers and BIM based scheduling. 2011. 95 (MSc). Civil \& Environmental Engineering, Worcester Polytechnic Institute, Worcester, UK.

HIJAZI, I. et al. IFC to CityGML Transformation Framework for Geo-Analysis: A Water Utility Network Case. 4th International Workshop on 3D Geo-Information. GENT, U. Ghent, Belgium 2009.

KARIMI, H. A.; AKINCI, B. CAD and GIS Integration. Florida, USA: Auerbach Publications, 2009. 247.

KHEMLANI, L. CORENET e-PlanCheck: Singapore's Automated Code Checking

System. AECbytes: Building the Future: Novacitynets: 8 p. 2005.

KOLBE, T. H. What is CityGML? , 2012. Disponível em: < http://www.citygml.org/index.php?id=1523 >. Acesso em: 06/01/2015.

KONRAD, C. P. Effects of Urban Development on Floods. U.S. GEOLOGICAL SURVEY, 2014. Disponível em: < http://pubs.usgs.gov/fs/fs07603/ >. Acesso em: 10/02/2015.

LAAKSO, M.; KIVINIEMI, A. The IFC standard - A review of history, development and standardization. Journal of Information Technology in Construction, v. 17, n. May 2012, p. 134-161, 2012. Disponível em: < http://www.itcon.org/data/works/att/2012 9.content.01913.pdf >. Acesso em: $13 / 05 / 15$. 
LIEBICH, T. What's new in IFC4? buildingSMART International Ltd. 2013

LIN, E. T. A.; FATT, C. T. Building Smart - A Strategy for Implementing BIM Solution in Singapore. Synthesis Journal. Singapore: 117-124 p. 2006.

MCROBERTS, P. How to Design and Build Future Sustainable Cities Using BIM. Guest Column, Fort Collins, EUA, 2010. Disponível em: < http://www.environmentalleader.com/2010/06/25/how-todesign-and-build-future-sustainable-cities-using-bim/ >. Acesso em: 15/03/2015.

MOTAWA, I.; CORRIGAN, W. Sustainable BIM-driven post-occupancy evaluation for buildings. CIC Start Feasibility Study, Glasgow, Scotland, p. 1-12, 2012. Disponível em: < http://www.cicstart.org/userfiles/file/FS-49-REPORT.PDF >. Acesso em: 12/03/2015.

NOUR, A. M. The Potential of GIS Tools in Strategic Urban Planning Process; as an Approach for Sustainable Development in Egypt. Journal of Sustainable Development, v. 4, n. 1, p. 15, 2011. Disponível em: < http://ccsenet.org/journal/index.php/jsd/article/view/8239/6820 >. Acesso em: 15/03/2015.

OLIVEIRA, M. R. Potential of Building Information Modeling (BIM) system. Innovative Developments in Design and Manufacturing: Advanced Research in Virtual and Rapid Prototyping. BARTOLO, P. J. D. S.;JORGE, M. A., et al. Leiria, Portugal: CRC Press: 695-700 p. 2009.

OLIVEIRA, P. A. D.; OLIVEIRA, M. P. G. Usos de um Sistema de Informação Geográfica em Cadastro Técnico Municipal: a experiência de Belo Horizonte. Informática Pública. Belo Horizonte: Empresa de Informática e Informação da Prefeitura de Belo Horizonte. 7: 67-84 p. 2005.

ORTH, D.; SILVA, E. Uso da tecnologia GIS em prefeituras brasileiras. III Simpósio Brasileiro de Ciências Geodésicas e Tecnologias da Geoinformação. Recife: UFPE: 1-6 p. 2010.

PRZYBYLA, J. The Next Frontier for BIM: Interoperability With GIS. Journal of Building Information Modeling. Washington, USA: The National Institute of Building Sciences: 14-18 p. 2010.

RECIFE, P. D. C. D. E-SIG - Informações Geográficas do Recife. Recife, 2010. Disponível em: < http://www.recife.pe.gov.br/ESIG/ >. Acesso em: 22/12/2014.

RUSCHEL, R. C. et al. O papel das ferramentas BIM de integração e compatrilhamento no processo de projeto na indústria da construção civil. REEC - Revista Eletrônica de Engenharia Civil. Goiânia, Brasil: Universidade Federal de Goiás. 7: 36-54 p. 2013.

SEBASTIAN, R. et al. Semantic BIM and GIS modelling for energy-efficient buildings integrated in a healthcare district. ISPRS 8th 3DGeolnfo Conference \& WG $11 / 2$ Workshop. ISPRS ANNALS OF THE PHOTOGRAMMETRY, R. S. A. S. I. S. Istanbul, Turkey. II-2 2013.

SILVA, M. V. D.; NEVES, T. D. F. Mapeamento Digital da Cidade do Recife - PE em SIRGAS 2000. IV Simpósio Brasileiro de Ciências Geodésicas e Tecnologias da Geoinformação. Recife: UFPE: 1-9 p. 2012.

SMARTCITIES. Using Geographic Information Systems to provide better e-services. A guide for municipalities from Smart Cities. SMARTCITIES. Edinburgh, UK: 52 p. 2011.

SUCCAR, B. Building Information Modelling: conceptual constructs and performance improvement tools. 2013. 370 (Doctor of Philosophy). School of Architecture and Built Environment, University of Newcastle, Newcastle, Australia.

UTIOME, E.; DROGEMULLER, R.; DOCHERTY, M. BIM-based lifecycle planning and specifications for sustainable cities of the future: A conceptual approach. International Council for Research and Innovation in Building and Construction. Lagos, Nigeria: CIB - Conseil International du Bâtiment: 580-589 p. 2014.

YEH, A. G. O. Urban planning and GIS. 2ª . New York: John Wiley \& Sons, 1999. 1297. 